\title{
Epidemiology and outcomes of out-of- hospital cardiac arrest according to suicide mechanism: a nationwide observation study
}

\author{
Soo Jin Kim ${ }^{1,2}$, Sang Do Shin ${ }^{3}$, Eui Jung Lee ${ }^{3}$, Young Sun $\mathrm{Ro}^{4}$, \\ Kyoung Jun Song ${ }^{3}$, Seung Chul Lee ${ }^{5}$; CardioVascular Disease \\ Surveillance (CAVAS) investigators \\ 'Seoul National University Graduate School of Medicine, Seoul, Korea \\ ${ }^{2}$ Seoul Metropolitan Fire Service Academy, Seoul, Korea \\ ${ }^{3}$ Department of Emergency Medicine, Seoul National University College of Medicine, Seoul, Korea \\ ${ }^{4}$ Laboratory of Emergency Medical Services, Seoul National University Hospital Biomedical Research \\ Institute, Seoul, Korea \\ ${ }^{5}$ Dongkuk University College of Medicine, Goyang, Korea
}

\begin{abstract}
Objective Suicide remains a serious, preventable public health problem. This study aims to describe the epidemiological characteristics associated with various suicide methods and to investigate outcomes after suicide-associated sudden cardiac arrest (S-SCA), stratified by different suicide attempt methods.

Methods An S-SCA database was constructed from ambulance run sheets and augmented by a review of hospital medical records from 2008 to 2010 in Korea. The cases with non-cardiac etiologies and suicide attempts were initially extracted. Suicide attempts were classified as hanging, poisoning, fall, and other. The primary end point was survival to discharge. Age- and sex-adjusted incidence rates were calculated for each suicide method. Adjusted odds ratios for outcome were calculated with adjustments for potential confounding variables.
\end{abstract}

Results A total 5,743 patients were analyzed as S-SCAs. The most common method of suicide attempt was hanging (58.7\%), followed by falls (17.6\%), poisoning (17.5\%), and others (5.8\%). The survival to discharge rates were $2.1 \%(n=119)$ overall, $2.4 \%$ in hanging, $2.4 \%$ in poisoning and $0.5 \%$ in fall, respectively. The age- and sex-adjusted incidence rates (male/female) per million persons was 32.7 (35.8/29.7) in 2008, 41.8 (46.0/37.7) in 2009, and 43.0 (50.1/36.0) in 2010. Compared with hanging, adjusted odds ratios (95\% confidence intervals) for survival to discharge was 1.05 (0.60 to 1.83 ) for poisoning and 0.08 (0.03 to 0.21 ) for falls.

Conclusion In this nationwide S-SCA cohort study from 2008 to 2010, the standardized incidence rate increased annually. However, the rate of survival to discharge remains very low.

Keywords Suicide; Heart arrest; Incidence; Outcome; Emergency medical services
elSSN: 2383-4625

Received: 3 March 2015

Revised: 15 April 2015

Accepted: 19 April 2015

Correspondence to: Sang Do Shin Department of Emergency Medicine, Seoul National University College of Medicine, 101 Daehak-ro, Jongno-gu, Seoul 110-744, Korea

E-mail:shinsangdo@medimail.co.kr

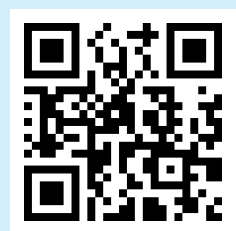

How to cite this article:

Kim SJ, Shin SD, Lee EJ, Ro YS, Song KJ, Lee SC; CardioVascular Disease Surveillance (CAVAS) investigators. Epidemiology and outcomes of out-of-hospital cardiac arrest according to suicide mechanism: a nationwide observation study. Clin Exp Emerg Med 2015;2(2):95-103.

This is an Open Access article distributed under the terms of the Creative Commons Attribution Non-Commercial License (http:// creativecommons.org/licenses/by-nc/3.0/). 


Capsule $\begin{aligned} & \text { What is already known } \\ & \text { Suicide is the most important public problem in Korea. Suicide rate has increased rapidly and many of victims are found } \\ & \text { at the scene with out-of-hospital cardiac arrest (OHCA) }\end{aligned}$
$\begin{aligned} & \text { What is new in the current study } \\ & \text { Outcomes are quite different according to suicide mechanism in OHCA. Hanging and poisoning to attempt suicide } \\ & \text { showed better outcomes by resuscitation effort than fall-down OHCA. }\end{aligned}$

\section{INTRODUCTION}

Suicide has long been recognized as a major public health and economic problem in various cultures. ${ }^{1,2}$ In 2002, an estimated 877,000 lives were lost worldwide through suicide, representing $1.5 \%$ of the total global burden of disease and more than 20 million disability-adjusted life-years (years of healthy life lost through premature death or disability). ${ }^{3}$ In contrast to the overall decrease in suicides among most industrialized countries over the last decade, Korea have experienced an increase in suicides. Between 1995 and 2006, Korea recorded the highest degree of suicide increase among the Organization for Economic Cooperation and Development countries, from 11.2 deaths per 100,000 to $21.5 .{ }^{4}$ Korea currently ranks first in the world, with a suicide rate of 33.4 for men and 18.7 for women in $2008 .{ }^{4}$

Sudden cardiac arrest (SCA) is normally the final process of successful suicides. Suicide-associated SCA (S-SCA) is most likely to be treated at the scene by emergency medical service (EMS) providers, transported by EMS, and subsequently managed by emergency department (ED) physicians as SCAs with a cardiac etiology. S-SCAs can provide valuable information on many different factors surrounding suicide attempts, such as the attempt method, time-course of the attempt, geospatial characteristics, presence or absence of witnesses, bystanders and bystander activity, EMS response time and services, and treatments initiated at the hospital. Previous studies described this information in SSCAs caused by drowning, poisoning, and hanging. ${ }^{5-7}$

This study aimed to describe the epidemiologic characteristics of SCA associated with suicide attempts and to investigate outcomes after S-SCA stratified by the different suicide attempt methods.

\section{METHODS}

The study was approved by the Institutional Board of Review at the study institution and financially supported by the Korea Centers for Disease Control and Prevention (2008 to 2011).

\section{Data source}

The Cardiovascular Disease Surveillance (CAVAS) database is a large-scale, nationwide, observational database of patients who have confirmed SCA in an out-of-hospital setting in Korea..$^{8}$ The database is population-based and contains EMS-assessed out-ofhospital cardiac arrests (OHCAs) derived from the entire population of the country. The cases were extracted from the National Emergency Management Agency's ambulance run sheets in which an OHCA was coded and additionally populated by hospital medical records review by the Korea Centers for Disease Control and Prevention. Cases from January 2008 to December 2010 were included. Trained medical record reviewers visited study hospitals and reviewed medical records to ensure the information related to risks and outcomes was complete, as per the Utstein guidelines for reporting cardiac arrest and resuscitation data. ${ }^{8}$

\section{Setting}

Korea has a population of approximately 48 million people, with a per capita gross domestic product of 20,591 US dollars and a life expectancy of 79.1 years (as of 2010). ${ }^{9}$ National statistics showed a $3.2 \%$ unemployment rate and a $2.3 \%$ crude divorce rate. Among all causes of mortality in Korea, suicide was the fifth leading cause. The total number of deaths per 100,000 people was 512 in 2010, and suicide accounted for 31.2 of these deaths. Mortality rates from suicide was also found to be more prevalent in younger and older populations. ${ }^{10}$

The Korean EMS system is single-tiered and provided by the government. A basic-to-intermediate level of ambulance services is operated by 16 provincial headquarters of the national fire department. The ambulance crews can perform cardiopulmonary resuscitation (CPR) at the scene and during transport, as well as provide care comparable to the intermediate emergency medical technician (EMT) level in the US. This includes starting intravenous fluids, endotracheal intubation, or laryngeal mask airway insertion under direct medical oversight.

All EDs are formally designated as level 1 to 3 by the government (20 regional EDs are designated level 1, 113 local EDs are 
designated level 2, and approximately 330 small EDs are designated level 3). The designation is based on several characteristics of the ED: human resources, essential instruments and equipment, and level of service available. Wilderness areas and isolated islands usually have no designated ED; therefore, most patients with an OHCA at these locations are transported to community health care centers or non-emergency hospitals (level 4, non-ED facilities). These facilities can perform CPR, followed by minimal primary care for patients with an OHCA. We collected information on OHCAs from these non-ED facilities. ${ }^{8}$

Currently, there is no specific EMS protocol for S-SCA, and EMTs cannot declare death or stop CPR in the field or during transport. However, they are able to seek medical directions from a physician via telephone when there is a suspicion of death, such as when decapitation, rigor mortis, decomposition, and dependent lividity are observed.

\section{Study population and data collection}

Eligible patients were S-SCA patients with a final outcome on record during the three years of the study period. The etiological classifications of an SCA were made by the physicians in the ED when the patients arrived and were presumed cardiac or non-cardiac according to the Utstein criteria. ${ }^{11}$ SCAs with non-cardiac etiology were further categorized using two criteria: the mechanism of attempted suicide and the intention, according to the international classification of external causes of injuries proposed by the World Health Organization. ${ }^{12}$ All non-cardiac etiologies were categorized as accidental, suicidal, homicidal, legal intervention, war/protest-associated, and other. In addition, all noncardiac etiologies were categorized by specific mechanism of injury: motor vehicle injury, fall, collision, piercing/stabbing/amputation, gunshot, burn, asphyxia, drowning, hanging, poisoning, and others. ${ }^{12}$ In this study, these categories are re-grouped under four major mechanisms: hanging, fall, poisoning, and other.

We collected demographic information regarding age $(\leq 15$, $16-64$, and $\geq 65$ years), sex, and primary rhythm as identified with electrocardiography. Rhythms were further categorized as shockable (ventricular fibrillation and pulseless ventricular tachycardia) or non-shockable (pulseless electrical activity, asystole, and unknown). Additional data included the presence of witnesses, location of the event (public, private, and unknown), indoor and outdoor buildings with stories where the event occurred, bystander CPR performed (based on a review of the medical records), response interval (time from call to ambulance arrival to the scene), EMS interval (time from call to arrival at hospital), certification level of the EMS provider (intermediate or basic), pre-hospital defibrillation, and the level of the ED (1 to 4). The onset time vari- ables were extracted from date and time of the emergency call, and the hour, week, and season were derived.

\section{Outcome measures}

The outcomes were classified by the rates of survival to admission, survival to discharge, and whether a good neurological outcome was achieved. The survival to hospital discharge rate was the primary end-point in this study. The survival to admission rate was defined as the cases with a return of spontaneous circulation that were admitted to the inpatient ward or the intensive care unit. A good neurological outcome was met when the surviving patient achieved a cerebral performance category of 1 or 2 . All outcomes were measured based on a review of medical records.

\section{Statistical analysis}

The distributions of categorical variables are reported as percentages. The continuous variables that were not distributed normally are presented as medians with interquartile ranges.

The age- and sex-adjusted incidence rates for all of the EMSassessed S-SCAs were calculated using the 2010 census data as the standard population (direct standardization method). The adjusted incidence rates were calculated according to the year, month, and by specific mechanism used.

The adjusted odds ratios (ORs) with 95\% confidence intervals (Cls) were calculated after adjusting using multivariable logistic regression analysis, including the variables as age, sex, primary electrocardiography type, etiology of arrest, year, witness, place of event, bystander CPR, response interval (from time of call to time of EMS arrival at the scene), EMS interval (from time of call to time of arrival at hospital), pre-hospital defibrillation, and ED level. To obtain the final model, we tested for interactions between exposure variables and potential covariates, assessed confounding factors, and performed precision level tests. The goodness of fit of the multivariable logistic regression model was tested using the Hosmer-Lemeshow (HL) test with chi-square analysis for calibration performance, and c statistics were measured to assess the discrimination performance of the final models. The analysis was conducted using SAS ver. 9.3 (SAS Institute, Cary, NC, USA).

\section{RESULTS}

\section{Demographics}

The total number of EMS-assessed SCAs was 64,155. Of these, the number analyzed as suicide-associated SCA in this study was 5,743 . SCAs from cardiac etiologies $(46,885)$ and non-suicidal and non-cardiac etiologies $(11,572)$ were excluded. Survival to 
admission and survival to discharge number (percentage) of the 5,743 patients analyzed was 631 (11.0\%) and 119 (2.1\%), respectively (Fig. 1). Of the 5,743 patients studied, the number analyzed increased every year. The most popular suicide mechanisms were hanging $(58.7 \%)$, followed by falling $(17.6 \%)$ and then poisoning (17.5\%). Other methods were used in $5.8 \%$ of cases. Drownings account 3.3\% for S-SCA patients, accounting for most of the cases in the "other" category, and were found to be more common in males and adults in non-metropolitan areas (Table 1).

\section{Age- and sex-adjusted incidence rates by mechanism}

Age- and sex-adjusted incidence rate (male/female) per million persons was 32.7 (35.8/29.7) in 2008, 41.8 (46.0/37.7) in 2009, and $43.0(50.1 / 36.0)$ in 2010. Hanging was the top method of attempting suicide (23.0 for total population, 24.9 for male subjects, and 21.1 for female subjects). Fall showed similar incidence rates between both sexes (Table 2).

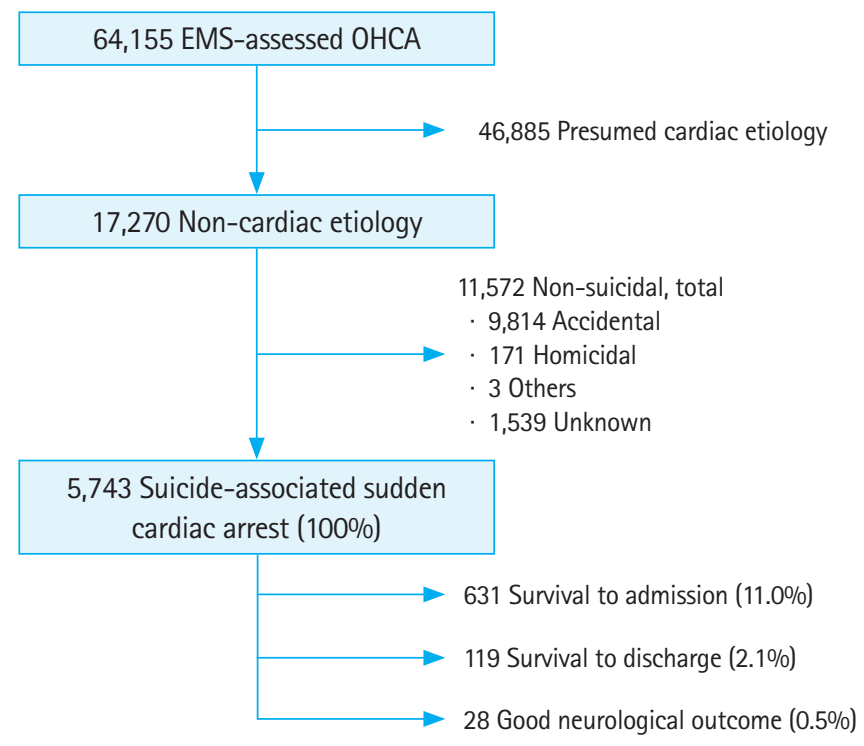

Fig. 1. Study population and outcomes. EMS, emergency medical service; OHCA, out-of-hospital cardiac arrest.

\section{Distribution of potential risk factors}

The distribution of potential risk factors by mechanism was described. Poisoning was more common in male subjects, elderly people, and subjects in non-metropolitan areas. Hanging was more common in male subjects and adults in metropolitan areas. Similarly, falls was found to be almost equal between sexes, and more common in adults in metropolitan areas. Response times and transport times were longer in the poisoning group than in other groups. Bystander CPR and defibrillation by EMS providers were extremely low in all mechanisms of attempted suicide. Comparable survival rates were found in hanging (2.4\%) and poisoning $(2.4 \%)$, whereas the fall group had a very low survival rate (0.5\%) (Table 3).

The study population was also described by month, weekday, and time of the attempted suicide. Mondays experienced the highest number of suicides regardless of mechanisms. Suicide rates reflecting the distribution of EMS call time for S-SCA patients were found to be lowest at the early morning (mid-night to midday) and peaked in the afternoon and evening hours (Fig. 2).

When we examined the data by year and month, the data showed a peak in hanging as the mechanism of suicide from October

Table 1. Distribution of mechanisms of suicide-associated sudden cardiac arrest by year

\begin{tabular}{lcccc}
\hline \multirow{2}{*}{ Mechanism } & \multicolumn{3}{c}{ Year } & Total \\
\cline { 2 - 4 } & 2008 & 2009 & 2010 & \\
\hline Total & $1,547(100)$ & $2,054(100)$ & $2,142(100)$ & $5,743(100)$ \\
Hanging & $884(57.1)$ & $1,204(58.6)$ & $1,280(59.8)$ & $3,368(58.7)$ \\
Poisoning & $313(20.2)$ & $344(16.7)$ & $347(16.2)$ & $1,004(17.5)$ \\
Fall & $259(16.7)$ & $372(18.1)$ & $380(17.7)$ & $1,011(17.6)$ \\
Other & $81(5.3)$ & $122(5.9)$ & $123(5.6)$ & $326(5.8)$ \\
Motor vehicle injury & $5(0.3)$ & $21(1)$ & $13(0.6)$ & $39(0.7)$ \\
Collision & $1(0.1)$ & $1(0)$ & $1(0)$ & $3(0.1)$ \\
Gunshot wound & $0(0)$ & $0(0)$ & $1(0)$ & $1(0)$ \\
Burn & $2(0.1)$ & $0(0)$ & $1(0)$ & $3(0.1)$ \\
Asphyxia & $7(0.5)$ & $18(0.9)$ & $7(0.3)$ & $32(0.6)$ \\
Drowning & $57(3.7)$ & $61(3)$ & $73(3.4)$ & $191(3.3)$ \\
Other & $4(0.3)$ & $1(0)$ & $0(0)$ & $5(0.1)$ \\
Unknown & $5(0.3)$ & $20(1)$ & $27(1.3)$ & $52(0.9)$ \\
\hline
\end{tabular}

Values are presented as $n(\%)$.

Table 2. Age- and sex-adjusted incidence of suicide-associated sudden cardiac arrest per million persons

\begin{tabular}{|c|c|c|c|c|c|c|c|c|c|c|c|c|}
\hline \multirow{2}{*}{ Mechanism } & \multicolumn{4}{|c|}{ Both sexes } & \multicolumn{4}{|c|}{ Male } & \multicolumn{4}{|c|}{ Female } \\
\hline & Total & 2008 & 2009 & 2010 & Total & 2008 & 2009 & 2010 & Total & 2008 & 2009 & 2010 \\
\hline Total & 39.4 & 32.7 & 41.8 & 43.0 & 44.1 & 35.8 & 46.0 & 50.1 & 34.5 & 29.7 & 37.7 & 36.0 \\
\hline Hanging & 23.0 & 18.5 & 24.4 & 25.7 & 24.9 & 19.2 & 26.0 & 29.1 & 21.1 & 17.9 & 22.9 & 22.3 \\
\hline Poisoning & 7.0 & 6.9 & 7.1 & 7.0 & 9.0 & 8.5 & 9.2 & 9.4 & 5.0 & 5.3 & 5.0 & 4.6 \\
\hline Fall & 6.9 & 5.4 & 7.6 & 7.6 & 6.8 & 5.5 & 7.1 & 7.8 & 6.9 & 5.3 & 8.0 & 7.5 \\
\hline Other & 2.5 & 1.9 & 2.7 & 2.7 & 3.4 & 2.6 & 3.7 & 3.8 & 1.5 & 1.2 & 1.8 & 1.6 \\
\hline
\end{tabular}

Age- and sex-adjusted incidence per million persons, using 2010 Korean census statistics. 
Table 3. Demographic findings of risk factors and outcomes

\begin{tabular}{|c|c|c|c|c|c|c|}
\hline \multicolumn{2}{|l|}{ Variables } & Total & Hanging & Poisoning & Fall & Other \\
\hline \multicolumn{2}{|l|}{ Total } & $5,743(100.0)$ & $3,368(100.0)$ & $1,004(100.0)$ & $1,011(100.0)$ & $360(100.0)$ \\
\hline \multirow[t]{2}{*}{ Year } & 2008 & $1,547(26.9)$ & $884(26.2)$ & $313(31.2)$ & $259(25.6)$ & $91(25.3)$ \\
\hline & 2010 & $2,142(37.3)$ & $1,280(38.0)$ & $347(34.6)$ & $380(37.6)$ & $135(37.5)$ \\
\hline \multirow[t]{2}{*}{ Sex } & Male & $3,194(55.6)$ & $1,806(53.6)$ & $646(64.3)$ & $496(49.1)$ & $246(68.3)$ \\
\hline & Female & $2,549(44.4)$ & $1,562(46.4)$ & $358(35.7)$ & $515(50.9)$ & $114(31.7)$ \\
\hline \multirow{2}{*}{ Age (yr) } & Adult (16-64) & $4,240(73.8)$ & $2,656(78.9)$ & $511(50.9)$ & $789(78.0)$ & $284(78.9)$ \\
\hline & Elderly ( $\geq 65$ ) & $1,431(24.9)$ & $686(20.4)$ & $491(48.9)$ & $181(17.9)$ & $73(20.3)$ \\
\hline \multirow[t]{3}{*}{ Primary ECG } & VF/pulseless VT & $43(0.7)$ & $25(0.7)$ & $10(1.0)$ & $2(0.2)$ & $6(1.7)$ \\
\hline & PEA & $148(2.6)$ & $58(1.7)$ & $23(2.3)$ & $54(5.3)$ & $13(3.6)$ \\
\hline & Asystole & $4,689(81.6)$ & $2,783(82.6)$ & $798(79.5)$ & $821(81.2)$ & $287(79.7)$ \\
\hline Community urbanization & Metropolitan & $2,344(40.8)$ & $1,423(42.3)$ & $266(26.5)$ & $453(44.8)$ & $202(56.1)$ \\
\hline & Unknown & $645(11.2)$ & $250(7.4)$ & $157(15.6)$ & $58(5.7)$ & $180(50.0)$ \\
\hline \multirow[t]{3}{*}{ Arrest witnessed } & Witnessed & $575(10.0)$ & $43(1.3)$ & $127(12.6)$ & $283(28.0)$ & $122(33.9)$ \\
\hline & Unwitnessed & $4,593(80.0)$ & $3,179(94.4)$ & 775 (77.2) & $481(47.6)$ & $158(43.9)$ \\
\hline & Unknown & $575(10.0)$ & $146(4.3)$ & $102(10.2)$ & $247(24.4)$ & $80(22.2)$ \\
\hline \multirow[t]{2}{*}{ Bystander CPR } & CPR & $150(2.6)$ & $129(3.8)$ & $8(0.8)$ & $8(0.8)$ & $5(1.4)$ \\
\hline & No CPR & $5,593(97.4)$ & $3,239(96.2)$ & 996 (99.2) & $1,003(99.2)$ & $355(98.6)$ \\
\hline \multicolumn{2}{|c|}{ Interval from call to EMS arrival (min) } & 5,657 & 3,322 & 994 & 985 & 356 \\
\hline & Median (IQR) & $6(5-9)$ & $6(5-9)$ & $8(5-12)$ & $6(4-7)$ & $7(5-11)$ \\
\hline & $0-4$ & $1,166(20.6)$ & $671(20.2)$ & $140(14.1)$ & $286(29.0)$ & $69(19.4)$ \\
\hline & $5-8$ & $1,102(19.5)$ & $1,772(53.3)$ & $409(41.1)$ & $536(54.4)$ & $155(43.5)$ \\
\hline & $9-12$ & 765 (13.5) & $552(16.6)$ & $210(21.1)$ & $125(12.7)$ & $63(17.7)$ \\
\hline & $13-16$ & $678(12.0)$ & $186(5.6)$ & $104(10.5)$ & $18(1.8)$ & $32(9.0)$ \\
\hline & $>32$ & $1,032(18.3)$ & $519(15.6)$ & $317(31.9)$ & $84(8.5)$ & $111(31.1)$ \\
\hline \multirow[t]{2}{*}{ Defibrillation by EMS } & EMS defibrillation & $60(1.0)$ & $33(1.0)$ & $4(0.4)$ & $13(1.3)$ & $10(2.8)$ \\
\hline & No defibrillation & $5,683(99.0)$ & 3,335 (99.0) & $1,000(99.6)$ & $998(98.7)$ & $350(97.2)$ \\
\hline \multirow[t]{4}{*}{ Level of destination hospital } & Level 1 ED & $591(10.3)$ & $300(8.9)$ & $87(8.7)$ & $147(14.5)$ & $57(15.8)$ \\
\hline & Level 2 ED & $2,498(43.5)$ & $1,455(43.2)$ & $353(35.2)$ & $514(50.8)$ & $176(48.9)$ \\
\hline & Level 3 ED & $2,214(38.6)$ & $1,354(40.2)$ & $456(45.4)$ & $298(29.5)$ & $106(29.4)$ \\
\hline & Non-ED facilities & $440(7.7)$ & $259(7.7)$ & $108(10.8)$ & $52(5.1)$ & $21(5.8)$ \\
\hline \multirow[t]{3}{*}{ Outcome } & Survival to admission & $631(11.0)$ & $472(14.0)$ & $104(10.4)$ & $12(1.2)$ & $43(11.9)$ \\
\hline & Survival to discharge & $119(2.1)$ & $79(2.3)$ & $24(2.4)$ & $5(0.5)$ & $11(3.1)$ \\
\hline & Good CPC 1 and 2 & $28(0.5)$ & $14(0.4)$ & $10(1.0)$ & $0(0.0)$ & $4(1.1)$ \\
\hline
\end{tabular}

Values are presented as $n(\%)$ unless otherwise indicated.

$I Q R$, interquartile range; ECG, electrocardiography; VF, ventricular fibrillation; VT, ventricular tachycardia; PEA, pulseless electrical activity; CPR, cardiopulmonary resuscitation; EMS, emergency medical service; ED, emergency department; CPC, Cerebral Performance Category scale.

2008 to December 2010. Before October 2008, the number of hangings was on average $<50$ per month, but this number increased to 213 in October 2008 and continued to be more than 100 per month after this point (Fig. 2).

\section{Main analysis}

Adjusted ORs (95\% Cls) for survival to admission rates compared with hanging was found to be 0.94 (0.72 to 1.24$)$ for poisoning, and 0.04 (0.02 to 0.08 ) for falls ( $\mathrm{HL}$ chi-square $=6.62, \mathrm{P}=0.58, \mathrm{c}$ statistics $=0.83$ ). With hanging as the index condition, adjusted 

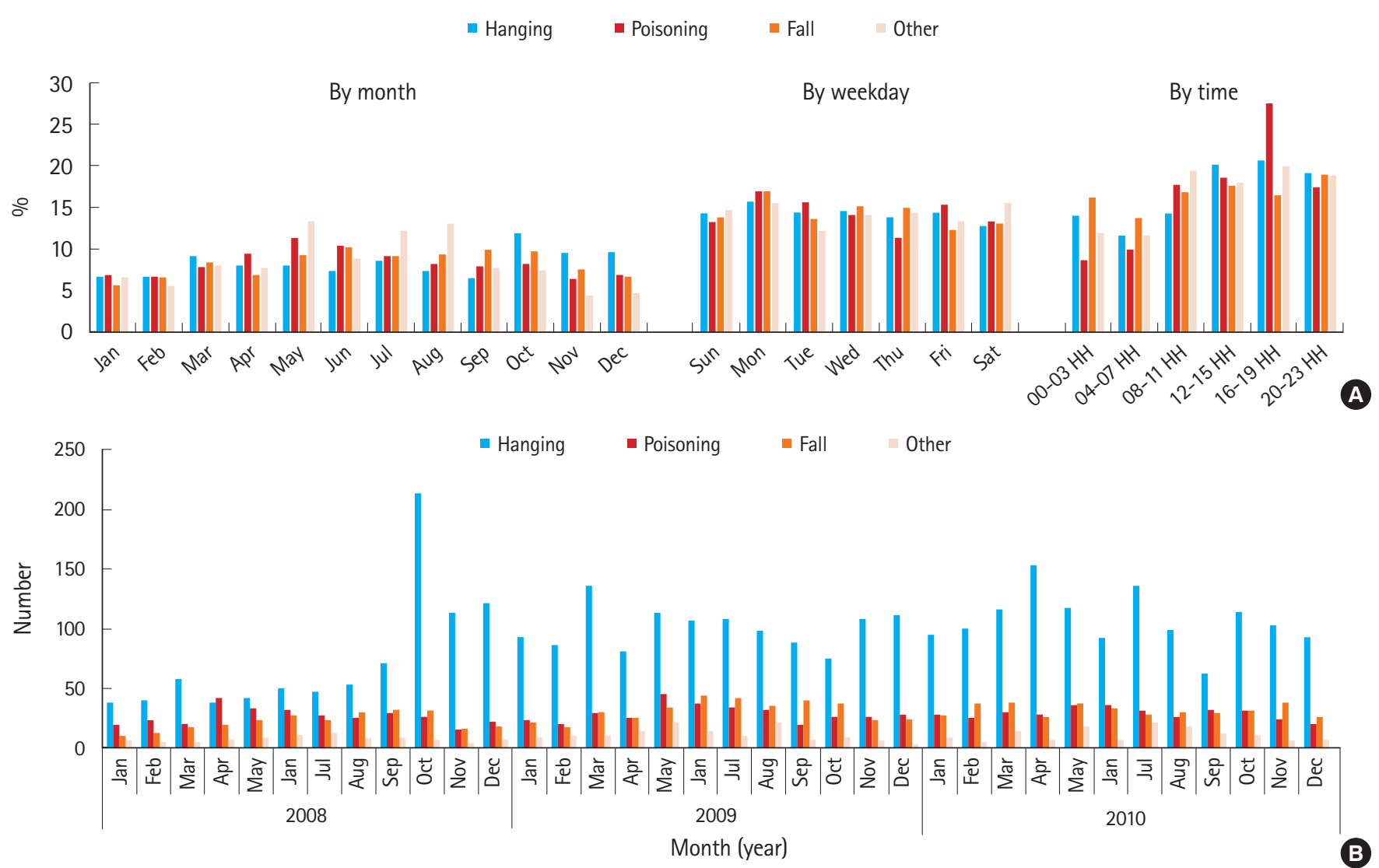

Fig. 2. Distribution and trend line of suicide-associated sudden cardiac arrests. (A) Distribution by month, weekday, and time of the event. (B) Monthly trend of suicide-associated sudden cardiac arrest by year and month. $\mathrm{HH}$, hour.

ORs (95\% Cls) for survival to discharge was 1.05 (0.60 to 1.83 ) for poisoning, and 0.08 ( 0.03 to 0.21 ) for falls ( $\mathrm{HL}$ chi-square $=3.68$, $\mathrm{P}=0.88$, c-statistics $=0.87)$. Adjusted ORs (95\% Cls) for good neurological outcomes compared with hanging was 3.31 (1.22 to 8.96) in poisoning. Of note, no case achieved a good neurological outcome in the fall category (HL chi-square $=10.98, P=0.09$, c-statistics $=0.93$ ) (Table 4).

\section{DISCUSSION}

Mechanisms for suicidal attempt are various, differ according to cultural background, and may change over time. ${ }^{2}$ People who have suicidal ideations generally seek isolated areas to avoid bystanders, use fast-acting suicide methods to reduce fear and pain, and ensure success via methods with a high rate of lethality. ${ }^{13-15}$

Suicide attempt patients are typically found in three different types of clinical situations: aborted suicide attempt, SCA, or death due to prolonged cardiac arrest (PCA). Aborted suicide is an incomplete suicide, while SCA and PCA are complete. Due to social, cultural, or religious backgrounds that regard suicide as a crime, conflicts within families, psychiatric disorders, and confidentiality, data on aborted suicides are extremely difficult to collect and analyze. ${ }^{16}$ Patients who experience PCA secondary to suicide are directly transported to the mortuary. The death certificates in these cases have limited information, making it difficult to adequately interpret the environment and characteristics of the suicide. ${ }^{17}$

This study is important for several reasons. An S-SCA database may be a good resource to understand various characteristics of suicide and to develop suicide prevention programs. Patients with S-SCA possess a host of information from the scene, which is a source of insight into primary prevention, EMS services, and hospital care and support for secondary prevention of suicide. $\mathrm{Na}-$ tionwide or community-based SCA registries, in particular OHCA registries, have been developed in both Asian and Western communities. ${ }^{18}$ Most SCA research focuses on SCA with presumed cardiac etiology or trauma-associated SCA rather than S-SCA. In Korea, which has a higher incidence of suicide, an S-SCA registry could prove to be a key resource in understanding the suicidal environment and method of choice; these data in turn may help to prevent suicide and improve resuscitation efforts and outcomes.

Suicide-associated SCA was observed to occur most commonly from hanging (58\%) and was responsible for $13.4 \%$ of the total 
Table 4. Multivariable logistic regression analysis for outcomes after suicide-associated sudden cardiac arrest by suicide mechanism

\begin{tabular}{|c|c|c|c|c|c|c|c|c|c|c|c|}
\hline \multirow{2}{*}{ Outcome } & \multirow{2}{*}{ Total } & \multirow{2}{*}{$\begin{array}{c}\text { Outcome } \\
\text { n (\%) }\end{array}$} & \multicolumn{3}{|c|}{ Unadjusted } & \multicolumn{3}{|c|}{ Adjusted $^{\text {a) }}$} & \multirow{2}{*}{$\begin{array}{l}\text { HL chi- } \\
\text { square }\end{array}$} & \multirow{2}{*}{ P-value } & \multirow{2}{*}{ c-statistic } \\
\hline & & & $\mathrm{OR}$ & & $\mathrm{Cl}$ & OR & & & & & \\
\hline Hanging (reference) & 3,368 & $472(8.2)$ & 1.00 & - & - & 1.00 & - & - & & & \\
\hline Poisoning & 1,004 & $104(1.8)$ & 0.71 & 0.57 & 0.89 & 0.94 & 0.72 & 1.24 & & & \\
\hline Fall & 1,011 & $12(0.2)$ & 0.07 & 0.04 & 0.13 & 0.04 & 0.02 & 0.08 & & & \\
\hline Total & 5,743 & $119(2.1)$ & - & - & - & - & - & - & 3.68 & 0.88 & 0.87 \\
\hline Hanging (reference) & 3,368 & $79(1.4)$ & 1.00 & - & - & 1.00 & - & - & & & \\
\hline Poisoning & 1,004 & $24(0.4)$ & 1.02 & 0.64 & 1.62 & 1.05 & 0.60 & 1.83 & & & \\
\hline Fall & 1,011 & $5(0.1)$ & 0.21 & 0.08 & 0.51 & 0.08 & 0.03 & 0.21 & & & \\
\hline Other & 360 & $11(0.2)$ & 1.31 & 0.69 & 2.49 & 0.68 & 0.29 & 1.57 & & & \\
\hline \multicolumn{12}{|c|}{ Good neurological recovery } \\
\hline Other & 360 & $4(0.1)$ & 2.69 & 0.88 & 8.22 & 1.51 & 0.35 & 6.52 & & & \\
\hline
\end{tabular}

$\mathrm{OR}$, odds ratio; $\mathrm{Cl}$, confidence interval; $\mathrm{HL}$, Hosmer-Lemeshow.

${ }^{a)}$ Adjusted for age, gender, primary electrocardiography type, etiology of arrest, year, witness, place of event, bystander cardiopulmonary resuscitation, response interval (from call to arrival at the scene), emergency medical service interval (from call to arrival at hospital), pre-hospital defibrillation, and emergency department level.

mortality rate from suicide of 32.1 per 100,000 persons, or 4.3 deaths per 100,000 persons. The survival to discharge rate was extremely low in all mechanisms of suicide and found to be even lower in falls compared with other methods of suicide in our nationwide S-SCA patients.

Our findings also demonstrated epidemiologic and geographic variability stratified by potential risk factors according to the different mechanisms of suicide. For example, non-metropolitan areas showed a relatively higher use of poisoning as a method of suicide rather than falls or hangings. This is in contrast to a separate study that demonstrated higher proportions of hanging in urban (18.0\%) compared to rural environments (14.9\%). ${ }^{19}$ In another population-based study from metropolitan Melbourne, Australia, hanging for suicide was common in the younger (median age 39 years) and male group (88\%). ${ }^{20}$ In another study from Taiwan, poisoning was found in higher rates in rural areas (32.1\%) than in urban environments (30.5\%). ${ }^{21}$ Our study showed hanging and poisoning was $57.7 \%$ and $73.5 \%$ in non-metropolitan areas, respectively. Poisoning has been a common method to attempt suicide in rural areas; however, our SCA registry showed much higher proportions of poisoning in rural areas than previously reported.

Our study suggests that different age groups tend to select different methods of attempting suicide. The young adult population (15 to 65 years) preferred hanging (62.6\%) over poisoning (12.1\%) or falls (18.6\%), whereas elderly people selected hanging (34.3\%).
More than half of the pediatric patients ( $<15$ years) selected falls $(56.9 \%)$ as the preferred method of suicide. One study showed much higher proportion of hanging (53.1\%) in young adult compared with poisoning (34.1\%) or falls (12.8\%). ${ }^{22}$ In another study from Hong Kong, carbon oxide from charcoal burning was an emerging method to commit suicide. ${ }^{23}$ Charcoal burning constituted $18.3 \%$ of all suicides in the study. Additionally, $88 \%$ of those that used charcoal burning were from the middle years ( 25 to 59 ) of life. The mechanism used to attempt or commit suicide has very strong sociocultural background and may change over time.

We found very interesting findings when analyzing monthly trends in this study. October 2008 showed a peak of hangings, which continued to the end of the observational period. We searched social events in this period and found two consecutive celebrity suicides on September 8 and October 2, 2008. In particular, the latter celebrity was a popular actress starring on a television drama in Korea. Major television news, newspaper reports, and internet media reports continued to report the event and method daily for a month. The first had attempted suicide via gas poisoning from charcoal burning, and the second used the hanging method. We believe the peak in October was due to copycat suicides and Werther's effect, ${ }_{1}^{24}$ as has been reported in many communities. ${ }^{25,26}$

We found very low rates of survival to discharge and neurological recovery in this study. Most previous studies reported outcomes on SCA with cardiac etiologies or trauma. From our previous reports, survival to discharge rates of SCA with presumed car- 
diac etiology transported by EMS was 3.7\% in 2008. ${ }^{27}$ Other reports on survival to discharge rates of SCA with an etiology of trauma was $1.0 \%{ }^{28}$ Hanging-associated SCA showed a very low survival to discharge rate $(0.5 \%)$ in another study with a small sample size. ${ }^{20}$ Our study demonstrated that lower survival to discharge rates in S-SCA is associated with lower witness rates (10.0\%), a private place $(81.9 \%)$, lower bystander CPR rate $(2.6 \%)$, asystolic rhythm (81.6\%), and more serious methods of suicide such as falls (17.6\%). Being in an isolated, private place for a suicide attempt is the main cause of extremely low witness rates and bystander CPR rates. This in turn leads to a high proportion of patients found by EMS to be in asystole and unable to be resuscitated. Our strategy for improving outcomes is to increase bystander CPR, even if the patient's suicide attempt is not witnessed and the patient is found at the scene after S-SCA. To improve bystander activity, we can encourage more specific population programs, such as dispatcher-assisted bystander CPR. ${ }^{29}$ However, more specific strategies for preventing suicide as well as improving bystander activity should be considered to remove the environmental conditions that favor suicide and to enable earlier detection in people at high risk of attempting suicide.

EMS-assessed and S-SCA registries can be extracted daily, as many communities have automated and electronic database systems in place. These registries will be very helpful for preventive strategies, because they allow for surveys and for the formation of daily and monthly suicide event trends. They may therefore assist in finding persons and locations at high risk of a suicide.

This study used a retrospective medical record review for measuring outcomes and risk factors. Additionally, there were many missing cases in some important variable categories. There were 1,539 cases without information on intentionality of the patient's suicide attempt. Although many observational studies on suicide have huge proportion of unknown intentionality, this unknown group can cause bias and underestimate the incidence rate by specific method. This study was performed in an EMS system with intermediate service level, which is a relatively lower level compared with those of North America and Europe. ${ }^{9}$ Therefore, our results may not be generalized to those communities. This study included cases in SCA status at the time when bystander witnessed or found the patients. If we added more cases of aborted suicide attempts or PCAs not requiring an ambulance transport, our results may be significantly changed.

In conclusion, from an EMS-assessed suicide-associated SCA registry, we found a suicide rate of 39.4 per million persons and a $2.1 \%$ rate of survival to discharge. Suicide by falls was associated with a significantly lower chance of survival to discharge as compared with suicide by hanging.

\section{CONFLICT OF INTEREST}

No potential conflict of interest relevant to this article was reported.

\section{ACKNOWLEDGMENTS}

This study was supported by the National Emergency Management Agency of Korea and the Korea Centers for Disease Control and Prevention.

\section{REFERENCES}

1. Yaniv G. Suicide intention and suicide prevention: an economic perspective. J Socio Econ 2001;30:453-68.

2. Mann JJ, Apter A, Bertolote J, et al. Suicide prevention strategies: a systematic review. JAMA 2005;294:2064-74.

3. World Health Organization. Country reports and charts [Internet]. Geneva: World Health Organization; 2005 [cited 2015 May 19]. Available from: http://www.who.int/mental_health/ suicide-prevention/en/.

4. Organization for Economic Cooperation and Development. OECD health data 2009. Paris: Organization for Economic Cooperation and Development; 2009.

5. Heming N, Serve $E$, Weiss $N$, et al. Drowning after falling from a medium-height bridge: multiple trauma victims. Prehosp Emerg Care 2012;16:356-60.

6. McLaughlin AM, Hardt J, McKay AP, Fitzpatrick GJ, Donnelly MB. Alcohol, drug misuse and suicide attempts: unrecognised causes of out of hospital cardiac arrests admitted to intensive care units. Ir J Med Sci 2009;178:29-33.

7. Deasy C, Bray J, Smith K, et al. Paediatric hanging associated out of hospital cardiac arrest in Melbourne, Australia: characteristics and outcomes. Emerg Med J 2011;28:411-5.

8. Shin SD, Suh GJ, Ahn KO, Song KJ. Cardiopulmonary resuscitation outcome of out-of-hospital cardiac arrest in low-volume versus high-volume emergency departments: an observational study and propensity score matching analysis. Resuscitation 2011;82:32-9.

9. Shin SD, Ong ME, Tanaka H, et al. Comparison of emergency medical services systems across Pan-Asian countries: a webbased survey. Prehosp Emerg Care 2012;16:477-96.

10. Korean Statistical Information Service. Statistical database [Internet]. Daejeon: Korean Statistical Information Service; 2014 [cited 2015 May 31]. Available from: http://kosis.kr/eng/ statisticsList/statisticsList_01List.jsp\#SubCont.

11. Jacobs I, Nadkarni V, Bahr J, et al. Cardiac arrest and cardio- 
pulmonary resuscitation outcome reports: update and simplification of the Utstein templates for resuscitation registries. A statement for healthcare professionals from a task force of the international liaison committee on resuscitation (American Heart Association, European Resuscitation Council, Australian Resuscitation Council, New Zealand Resuscitation Council, Heart and Stroke Foundation of Canada, InterAmerican Heart Foundation, Resuscitation Council of Southern Africa). Resuscitation 2004;63:233-49.

12. Scott D, Harrison J, Purdie D, et al. The properties of the International Classification of the External Cause of Injury when used as an instrument for injury prevention research. Inj Prev 2006;12:253-7.

13. Kim SY, Kim MH, Kawachi I, Cho Y. Comparative epidemiology of suicide in South Korea and Japan: effects of age, gender and suicide methods. Crisis 2011;32:5-14.

14. Scocco P, de Girolamo G, Vilagut G, Alonso J. Prevalence of suicide ideation, plans, and attempts and related risk factors in Italy: results from the European Study on the Epidemiology of Mental Disorders: World Mental Health study. Compr Psychiatry 2008;49:13-21.

15. Page RM, West JH. Suicide ideation and psychosocial distress in sub-Saharan African youth. Am J Health Behav 2011;35: 129-41.

16. Van Orden KA, Witte TK, Cukrowicz KC, Braithwaite SR, Selby EA, Joiner TE Jr. The interpersonal theory of suicide. Psychol Rev 2010;117:575-600.

17. Barber ME, Marzuk PM, Leon AC, Portera L. Aborted suicide attempts: a new classification of suicidal behavior. Am J Psychiatry 1998;155:385-9.

18. Berdowski J, Berg RA, Tijssen JG, Koster RW. Global incidences of out-of-hospital cardiac arrest and survival rates: systematic review of 67 prospective studies. Resuscitation 2010;81: 1479-87.

19. Obafunwa JO, Busuttil A. A review of completed suicides in the Lothian and Borders Region of Scotland (1987-1991). Soc
Psychiatry Psychiatr Epidemiol 1994;29:100-6.

20. Deasy C, Bray J, Smith K, Bernard S, Cameron P; VACAR Steering Committee. Hanging-associated out-of-hospital cardiac arrests in Melbourne, Australia. Emerg Med J 2013;30:38-42.

21. Chang SS, Lu TH, Sterne JA, Eddleston M, Lin JJ, Gunnell D. The impact of pesticide suicide on the geographic distribution of suicide in Taiwan: a spatial analysis. BMC Public Health 2012;12:260.

22. Yip PS, Caine ED, Kwok RC, Chen YY. A decompositional analysis of the relative contribution of age, sex and methods of suicide to the changing patterns of suicide in Taipei City, 20042006. Inj Prev 2012;18:187-92.

23. Law CK, Yip PS, Caine ED. The contribution of charcoal burning to the rise and decline of suicides in Hong Kong from 19972007. Soc Psychiatry Psychiatr Epidemiol 2011;46:797-803.

24. Jeong J, Shin SD, Kim H, Hong YC, Hwang SS, Lee EJ. The effects of celebrity suicide on copycat suicide attempt: a multicenter observational study. Soc Psychiatry Psychiatr Epidemiol 2012;47:957-65.

25. Cheng AT, Hawton $K$, Lee $C T$, Chen $T H$. The influence of media reporting of the suicide of a celebrity on suicide rates: a population-based study. Int J Epidemiol 2007;36:1229-34.

26. Phillips DP. The influence of suggestion on suicide: substantive and theoretical implications of the Werther effect. Am Sociol Rev 1974;39:340-54.

27. Ro YS, Shin SD, Song KJ, et al. A trend in epidemiology and outcomes of out-of-hospital cardiac arrest by urbanization level: a nationwide observational study from 2006 to 2010 in South Korea. Resuscitation 2013;84:547-57.

28. Ro YS, Shin SD, Song KJ, et al. A comparison of outcomes of out-of-hospital cardiac arrest with non-cardiac etiology between emergency departments with low- and high-resuscitation case volume. Resuscitation 2012;83:855-61.

29. White $L$, Rogers J, Bloomingdale $M$, et al. Dispatcher-assisted cardiopulmonary resuscitation: risks for patients not in cardiac arrest. Circulation 2010;121:91-7. 